Z. klin. Chem. u. klin. Biochem.

8. Jg., S. $65-68$, Januar 1970

\title{
Wasser- und Elektrolytverteilung in einigen Organen von Mg-arm ernährten Ratten
}

\author{
Von Th. GüNTHER \\ Aus dem Pbysiologisch-Chemischen Institut der Freien Universität Berlin (Direktor: Prof. Dr. Dr. E. Schïtte)
}

(Eingegangen am 29. September 1969)

An jungen männlichen Albinoratten wurde nach 38 tägiger $\mathrm{Mg-armer}$ Ernährung in Leber, Niere, Herz und Skelettmuskel der $\mathrm{H}_{2} \mathrm{O}-$, $\mathrm{Na}-$, K- und Mg-Gehalt und in Leber und Skelettmuskel zusätzlich die Größe der extra- und intrazellulären Flüssigkeit sowie die intrazelluläre $\mathrm{Na}$ - und K-Konzentration bestimmt.

Im Skelettmuskel nahm die extrazelluläre Flüssigkeit zu und die intrazelluläre K-Konzentration ab. In der Leber verringerte sich die extrazelluläre Flüssigkeit und die intrazelluläre K-Konzentration, die intrazelluläre Na-Konzentration stieg an.

Die Ergebnisse werden mit einer Abnabme des aktiven Alkaliionentransportes erklärt und diskutiert.

\section{Electrolyte distribution in some organs of rats with a dietary deficiency of $\mathrm{Mg}$}

Young male albino rats were fed a $\mathrm{Mg}$-deficient diet for 38 days. The content of $\mathrm{H}_{2} \mathrm{O}, \mathrm{Na}, \mathrm{K}$ and $\mathrm{Mg}$ in kidney and heart, in the liver and skeletal muscle, the volume of the extra-and intracellular fluid, and the concentration of intracellular $\mathrm{Na}$ and $\mathrm{K}$ were also determined. The extracellular fluid increased in the skeletal muscle and the intracellular concentration of $\mathrm{K}$ decreased. In the liver, the extracellular fluid and the intracellular concentration of $\mathrm{K}$ were decreased and the intracellular concentration of $\mathrm{Na}$ was increased.

The results are explained and discussed on the basis of a decrease in the active transport of alkali ions.

Bei Zellen von E. coli, deren Mg-Gehalt nach Wachsen in $\mathrm{Mg}$-armen Medium von etwa $20 \mathrm{mMol} / \mathrm{kg}$ auf $5 \mathrm{mMol} / \mathrm{kg}$ abgenommen hatte (1), war der aktive K-Transport in die Zelle erheblich vermindert (2). An tierischen Organen dagegen läßt sich keine signifikante oder nur eine geringfügige Abnahme des $\mathrm{Mg}$-Gehaltes erreichen, besonders wenn man junge, noch wachsende Tiere "Mgfrei“c, d.h. Mg-arm ernährt (3, 4, 5, Ubbersicht s. 6). Auch hierbei wurde eine Abnahme des K-Gehaltes in einigen Organen, hauptsächlich im Muskel, aber nicht in allen untersuchten Organen gefunden $(3,5)$.

Aus den bisherigen Untersuchungen an tierischen Geweben lassen sich jedoch keine Aussagen über das Verhalten der intrazellulären $\mathrm{Na}$ - und K-Konzentrationen und damit über das Verhalten des aktiven Transportes machen, da die Größe der extrazellulären Flüssigkeit nicht ermittelt wurde. Lediglich WHANG und Mitarbeiter (4) bestimmten im Skelettmuskel den Cl-Raum, der aber für die extrazelluläre Flüssigkeit $z u$ hohe Werte ergibt und kein richtiges $\mathrm{Maß}$ für die Größe der extrazellulären Flüssigkeit darstellt (7).

In der vorliegenden Mitteilung wurde deshalb zusätzlich die Größe der extrazellulären Flüssigkeit in Leber und Muskel als Rohrzuckerverteilungstaum und mit ihrer Hilfe die Verteilung des $\mathrm{H}_{2} \mathrm{O}, \mathrm{Na}$ und $\mathrm{K}$ zwischen intra- und extrazellulärem Raum sowie die intrazelluläre Alkaliionenkonzentration bestimmt.

\section{Methodik}

Wir benutzten männliche Albinoratten (Wistar) mit einem durchschnittlichen Gewicht von $114 \pm 6 \mathrm{~g} .28$ Tiere erhielten $\mathrm{Mg}$ armes, 9 Kontrolltiere Mg-reiches Futter und bidest. Wasser. Das Futter war wie folgt zusammengesetzt: $200 \mathrm{~g}$ Casein (Merck, nach Hammarsten), $100 \mathrm{~g}$ Olivenöl, $660 \mathrm{~g}$ Rohrzucker, $1 \mathrm{~g}$ einer Mischung von Spurenelementen (Zusammensetzung der Salz- mischungen nach MACINTYRE und Davidson (3)) sowie $40 \mathrm{~g}$ der Salzmischung C (3) für die Kontrolltiere bzw. 36,7 g der Salzmischung $D$ (3) für die $\mathrm{Mg}$-arm ernährten Tiere.

An Vitaminen wurde zugesetzt:

$\begin{array}{lr}\text { Vitamin A } & 12000 \mathrm{I} . \mathrm{E} . \\ \text { Thiaminmononitrat } & 18 \mathrm{mg} \\ \text { Riboflavin } & 18 \mathrm{mg} \\ \text { Pyridoxin } \cdot \mathrm{HCl} & 24 \mathrm{mg} \\ \text { Cyanokobalamin } & 12 \mu \mathrm{g} \\ \text { Ascorbinsäure } & 600 \mathrm{mg} \\ \alpha \text {-Tocopherolacetat } & 24 \mathrm{mg} \\ \text { Vitamin D } & 600 \mathrm{I} . \mathrm{E} . \\ \text { Vitamin K (Synkavit) } & 5 \mathrm{mg} \\ \text { Nicotinsäureamid } & 120 \mathrm{mg} \\ \text { Folsäure } & 3 \mathrm{mg} \\ \text { Ca-Panthotenat } & 48 \mathrm{mg} \\ \text { Biotin } & 2 \mathrm{mg} \\ \text { Inosit } & 200 \mathrm{mg} \\ \text { p-Aminobenzoesäure } & 200 \mathrm{mg} \\ \text { Cholinchlorid } & 2 \mathrm{~g}\end{array}$

Die Nahrung enthielt (nach eigener Analyse) Na: $63 \mathrm{mMol} / \mathrm{kg}$, $\mathrm{K}: 149 \mathrm{mMol} / \mathrm{kg}, \mathrm{Ca}: 145 \mathrm{mMol} / \mathrm{kg}, \mathrm{Mg}: 16 \mathrm{mMol} / \mathrm{kg}$ (Mg-reich) bzw. 0,5 mMol $/ \mathrm{kg}$ (Mg-arm).

Diese Diät wurde 38 Tage lang verfüttert. Während dieser Zeit starben 15 der $\mathrm{Mg}$-arm ernährten Tiere. Am Versuchsende (nach 38 Tagen) wurde den Tieren zur Bestimmung der extrazellulären Flüssigkeit $1 \mathrm{~m} / 14$ proz. Rohrzuckerlösung in eine Schwanzvene injiziert. $15 \mathrm{Min}$. danach wurde den Tieren in Äthernarkose mit einer Injektionsspritze Blut aus dem Herzen abgesaugt, anschlieBend wurden identische Herz-, Leber-, Nieren- und Muskel(Oberschenkel)-Proben entnommen und von anbaftendem Blut, Binde- und Fettgewebe befreit. Die Gewebeproben wurden gewogen, in flüssiger Luft eingefroren und im Vakuum über Silicagel bei etwa 0,5 Torr gefriergetrocknet.

In den Seren wurde die $\mathrm{Na}-, \mathrm{K}-, \mathrm{Mg}$-, $\mathrm{Ca}$ - und die Rohrzuckerkonzentration, von den Geweben der Wassergehalt, die extrazelluläre Flüssigkeit, der $\mathrm{Na}$-, $\mathrm{K}$ - und $\mathrm{Mg}$-Gehalt, wie früher ausführlich beschrieben $(7,8)$, bestimmt. Die Ca-Konzentration im Serum wurde durch photometrische Titration mit EDTA ermittelt (9). 
Die Analysenergebnisse der Organe wurden auf Feuchtgewicht bezogen. Vom Gesamt Na- und K-Gehalt wurde zur Ermittlung der intrazellulären Werte der extrazelluläre $\mathrm{Na}$ - und $\mathrm{K}$-Gehalt in Leber und Muskel abgezogen. Der extrazelluläre Na- und KGehalt wurde aus dem extravasalen und intravasalen Anteil, dessen Verhältnis mit 1:3 angenommen wurde, unter Berücksichtigung des Donrianfaktors $(0,95)$ ermittelt. Zur Ermittlung der intrazellulären $\mathrm{Na}-\mathrm{K}-\mathrm{Konzentration} \mathrm{wurde} \mathrm{durch} \mathrm{die} \mathrm{Menge} \mathrm{des}$ intrazellulären Wassers geteilt (genaue Berechnung s. $(7,8)$ ).

\section{Ergebnisse und Diskussion}

Nach etwa 10 Tagen stelllten sich bei den Mg-arm ernährten Tieren die typischen von allen Untersuchern beschriebenen Mg-Mangelerscheinungen, Vasodilatation und Hyperämie der Ohren, ein, die etwa eine Woche andauern und woran sich Nekrosen an den Ohrrändern anschließen. Gleichzeitig treten bei einigen Tieren Ödeme an den Pfoten auf $(3,4,5,6)$.

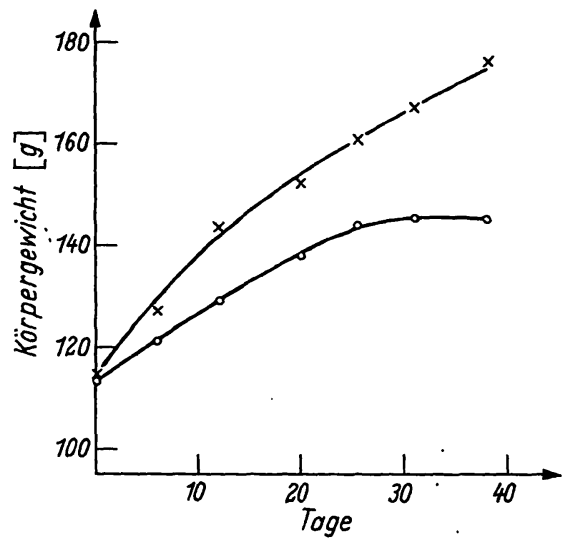

Abb. 1 Wachstumskurve von Magnesium-reich $(x)$ und Magnesium-arm (o)

Die Mg-arm ernährten Tiere zeigten anfangs eine geringere Gewichtszunahme als die Kontrolltiere. Von der vierten Woche an bis zum Versuchsende nahm ihr Gewicht nicht mehr zu (Abb. 1). Bei längerer Versuchsdauer kann sogar Gewichtsverlust eintreten $(3,5)$.

Tab. 1

Na-, K-, Ca- und Mg-Konzentration im Serum (mMol/l)

\begin{tabular}{ccccc}
\hline & $\mathrm{Na}$ & $\mathrm{K}$ & $\mathrm{Ca}$ & $\mathrm{Mg}$ \\
\hline Kontrolltiere & $147,4 \pm 0,7$ & $7,01 \pm 0,07$ & $2,50 \pm 0,07$ & $0,90 \pm 0,04$ \\
Mg-arm & $146,0 \pm 1,2$ & $6,79 \pm 0,21$ & $2,70 \pm 0,08$ & $0,23 \pm 0,02$ \\
\hline
\end{tabular}

Nach 38 tägiger $\mathrm{Mg}$-armer Ernährung war gegenüber der Kontrollgruppe die Na- und K-Konzentration im Serum unverändert, die $\mathrm{Mg}$-Ǩ onzentration von 0,90 auf $0,23 \mathrm{mMol} / l$ abgesunken und die Ca-Konzentration von 2,50 auf 2,70 mMol / l angestiegen (Tab. 1).

Während der 38 tägigen $\mathrm{Mg}$-armen Ernährung nahm der $\mathrm{Mg}-$ Gehalt in der.Niere um 5\%, in der Leber um $10 \%$, im Herzmuskel um 14\%, und im Skelettmuskel um $21 \%$ ab (Tab. 3). Die Abnahme des Mg-Gehaltes in den Geweben ist also gegenüber der Abnahme im Serum sehr gering. Daraus folgt, daß die Serum-Mg-Konzentration ein empfindlicher Indikator für einen $\mathrm{Mg}$-Mangel des Organismus ist und eine Substitution mit $\mathrm{Mg}$ nur bei verminderter Serum-Mg-Konzentration sinnvoll ist. Der K-Gehalt verhielt sich ähnlich wie das $\mathrm{Mg}$. In der Niere fanden wir keine signifikante Änderung, in der Leber und im Herzmuskel eine geringe und im Skelettmuskel die größte Abnahme (Tab. 2).

Der Na-Gehalt verhielt sich reziprok: In der Niere änderte er sich nicht signifikant, in Leber und Herz nahm er wenig und in der Skelettmuskulatur stark zu.

Ein analoges Verhalten zeigte auch der Wassergehalt. In Niere und Herz blieb er konstant, in der Leber nahm er etwas und im Skelettmuskel am stärksten.zu (Tab. 2). Die Größe der extrazellulären Flüssigkeit konnte wegen der dazu benötigten Substanzmenge nur in Leber und Skelettmuskel ermittelt werden. In der Leber nahm sie von $22,4 \%$ auf $15,0 \%$ ab, im Skelettmuskel von $9,9 \%$ auf $22,0 \%$ zu (Tab. 2).

Tab. 3

Na-, $\mathrm{K}$ - und $\mathrm{Mg}$-Gehalt in Rattenorganen bezogen auf Feuchtigkeit

\begin{tabular}{cccc}
\hline & $\mathrm{Na}$ & $\begin{array}{c}\mathrm{K} \\
\mathrm{mMol} / \mathrm{kg}\end{array}$ & $\begin{array}{c}\mathrm{Mg} \\
\mathrm{mMol} / \mathrm{kg} \\
\mathrm{mMol} / \mathrm{kg}\end{array}$ \\
\hline Kontrolltiere & & & \\
Herz & 49,1 & $\mathbf{7 1 , 5}$ & $\mathbf{8 , 5 8}$ \\
Leber & 31,7 & 89,0 & 10,9 \\
Niere & 65,7 & 62,1 & 8,61 \\
Muskel & $\mathbf{3 0 , 9}$ & $\mathbf{9 6 , 5}$ & $\mathbf{1 2 , 0}$ \\
Mg-arm & & & \\
Herz & 53,9 & 68,5 & $\mathbf{7 , 3 6}$ \\
Leber & 35,7 & 85,1 & 9,85 \\
Niere & 66,5 & 64,5 & $\mathbf{8 1 7}$ \\
Muskel & $\mathbf{4 3 , 2}$ & $\mathbf{7 5 , 4}$ & $\mathbf{9 , 4 4}$ \\
\hline
\end{tabular}

Die starke Zunahme der extrazellulären Flüssigkeit im Muskel läßt sich, wie"gleichzeitig ausgeführte elektronenmikroskopische Untersuchungen ergaben (10), auf eine geringe Vergrößerung des interstitiellen Raumes und auf eine Erweiterung des transversalen Tubulus-

Tab. 2

Analysen der gefriergetrockneten Organe

\begin{tabular}{|c|c|c|c|c|c|}
\hline & $\mathrm{H}_{2} \mathrm{O} \%$ & $\begin{array}{l}\text { extrazelluläre } \\
\text { Flüssigkeit \% }\end{array}$ & $\begin{array}{c}\text { Na mMol/kg } \\
\text { Trockensubstanz }\end{array}$ & $\underset{\text { Trockensubstanz }}{\mathrm{K} \mathrm{mMol} / \mathrm{kg}}$ & $\begin{array}{c}\mathrm{Mg} \mathrm{mMol} / \mathrm{kg} \\
\text { Trockensubstanz }\end{array}$ \\
\hline \multicolumn{6}{|c|}{ Kontrolltiere } \\
\hline Herz & $75,46 \pm 0,24$ & - & $200,0 \pm 7,2$ & $290,9 \pm 6,3$ & $34,9 \pm 0,5$ \\
\hline Leber & $68,33 \pm 0,27$ & $22,4 \pm 2,2$ & $99,8 \pm 2,4$ & $280,5 \pm 2,5$ & $34,3 \pm 0,9$ \\
\hline Niere & $76,34 \pm 0,37$ & 工 & $277,3 \pm 6,6$ & $261,9 \pm 6,6$ & $36,3 \pm 0,7$ \\
\hline Muskel & $73,83 \pm 0,32$ & $9,9 \pm 0,4$ & $117,8 \pm 2,2$ & $368,3 \pm 11,0$ & $45,9 \pm 1,0$ \\
\hline \multicolumn{6}{|l|}{$M g$-arm } \\
\hline Herz & $75,20 \pm 0,22$ & - & $217,3 \pm 9,9$ & $276,1 \pm 6,1$ & $29,7 \pm 1,4$ \\
\hline Leber & $69,80 \pm 0,34$ & $15,0 \pm 1,2$ & $118,7 \pm 3,7$ & $281,9 \pm 6,0$ & $32,6 \pm 0,6$ \\
\hline Niere & $76,01 \pm 0,41$ & 二 & $277,6 \pm 6,0$ & $268,2 \pm 4,4$ & $34,0 \pm 0,6$ \\
\hline Muskel & $76,40 \pm 0,28$ & $22,0 \pm 1,6$ & $182,9 \pm 7,6$ & $319,6 \pm 7,3$ & $40,0 \pm 2,1$ \\
\hline
\end{tabular}


systems zurückführen, das mit der extrazellulären Flüssigkeit in Verbindung steht $(11,12,13,14)$ und das sich auch unter anderen Bedingungen, z. B. Behandlung mit Methylthiouracil, erweitert (15). Möglicherweise sind auch die im $\mathrm{Mg}$-Mangel vermehrt auftretenden submembranösen Cysternen an der Zunahme der extrazellulären Flüssigkeit beteiligt (10). : $;$

Die Kenntnis der Größe der extrazellulären'Flüssigkeit gestattet es, die Menge des intrazellulären Wassers und

Tab. 4

Extrazellulärer (EZ) und intrazellulärer (IZ) Na- und K-Gehalt sowie intrazelluläre $\mathrm{Na}$ - und $\mathrm{K}$-Konzentration in den Organen der normalen und $\mathrm{Mg}$-arm ernährten Ratten

\begin{tabular}{|c|c|c|c|c|c|c|}
\hline & 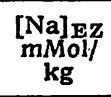 & $\begin{array}{c}{[\mathrm{Na}] \mathrm{TIz}} \\
\mathbf{m M g} /] \\
\mathbf{k g}\end{array}$ & $\begin{array}{c}{[\mathrm{Na}] \mathrm{gz}} \\
\text { mMll/ } \\
l\end{array}$ & 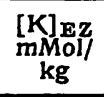 & 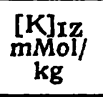 & $\begin{array}{c}{[\mathrm{K}] \mathrm{Iz}} \\
\text { mMoll } \\
\boldsymbol{l}\end{array}$ \\
\hline 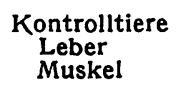 & $\begin{array}{l}31,7 \\
14,0\end{array}$ & $\begin{array}{c}0 \\
16,9\end{array}$ & $\begin{array}{c}0 \\
26,4\end{array}$ & $\begin{array}{l}1,51 \\
0,66\end{array}$ & $\begin{array}{l}87,5 \\
95,8\end{array}$ & \\
\hline $\begin{array}{l}\text { Ig-arm } \\
\text { Leber } \\
\text { Muskel }\end{array}$ & $\begin{array}{r}21,6 \\
30,9\end{array}$ & $\begin{array}{l}14,1 \\
12,3\end{array}$ & $\begin{array}{l}25,5 \\
22,7\end{array}$ & $\begin{array}{l}0,98 \\
1,43\end{array}$ & $\begin{array}{l}84,1 \\
74,0\end{array}$ & $\begin{array}{l}152 \\
136\end{array}$ \\
\hline
\end{tabular}

daraus, wenn man annimmt, daß das intrazelluläre Wasser vollständig zum Lösen des gesamten intrazellulären $\mathrm{Na}$ bzw. $\mathrm{K}$ zur Verfügung steht, die intrazellulären Alkali-Konzentrationen zu berechnen.

Der vermehrten extrazellulären Flüssigkeit im Muskel entspricht ein vergrößerter Na-Gehalt. Die Berechnung des extra- und intrazellulären $\mathrm{Na}$-Gehaltes sowie der intrazellulären $\mathrm{Na}$-Konzentration zeigt, daß die $\mathrm{Zu}-$ nahme des Na-Gehaltes nur extrazellulär erfolgt (s. Tab. 4). Die auf gleiche Weise erhaltene intrazelluläre $\mathrm{K}-$ Konzentration im Muskel ist bei den $\mathrm{Mg}$-arm ernährten Tieren von $150 \mathrm{mMol} / \mathrm{l}$ auf $136 \mathrm{mMol} / \mathrm{l}$ abgesunken (Tab. 4).

Die intrazelluläre K-Konzentration in der Leber hat noch stärker, nämlich von 194 auf $152 \mathrm{mMol} / \mathrm{l}$ abgenommen. Die intrazelluläre Na-Konzentration in der Leber verhält sich entgegengesetzt. Sie steigt von 0 bei den Kontrolltieren auf $25,5 \mathrm{mMol} / l$ bei den Versuchstieren an (Tab. 4).

Das Verhalten der intrazellulären K-Konzentration in Leber und Muskel läßt sich mit einer Abnahme des KInfluxes oder Zunahme des K-Effluxes erklären. Das reziproke Verhalten des $\mathrm{Na}$ in der Leber deutet auf eine Kopplung des $\mathrm{K}$ - und Na-Transportes. Die Ergebnisse lassen den Schluß zu, daß der aktive Na-K-Transport durch die Zellmembran, der für die unterschiedliche $\mathrm{Na}$ - und K-Verteilung $\mathrm{zwischen}$ intra- und extrazellulärer Flüssigkeit verantwortlich ist, bei den Mg-Mangeltieren verringert ist. Hiermit stimmt überein, $\mathrm{da} B$ der $\mathrm{K}$-Verlust bei $\mathrm{Mg}$-Mangeltieren nicht durch eine verminderte Nierenleistung erklärt werden konnte (16) und auch bei erhöhter K-Zufuhr mit der Nahrung erfolgte (4). Eine Abnahme des aktiven Alkaliionentransportes bei $\mathrm{Mg}$-arm ernährten Tieren enspräche auch der Abnahme des aktiven K-Transportes, die wir früher bei $\mathrm{Mg}$-arm gewachsenen Zellen von $E$. coli gefunden haben (2).

Die Mg-Werte (Tab. 3) wurden nicht in gleicher Weise behandelt wie die $\mathrm{Na}$ - und $\mathrm{K}$-Werte, denn der extra- zelluläre $\mathrm{Mg}$-Anteil beträgt gegenüber dem intrazellulären $\mathrm{Mg}$ nur etwa $1 \%$ und kann daher vernachlässigt werden. Eine berechnete intrazelluläre Mg-Konzentration würde nichts über das Verhalten der intrazellulären $\mathrm{Mg}$-Ionenkonzentration und des $\mathrm{Mg}$-Transportes aussagen, denn das $\mathrm{Mg}$ in der Zelle ist zu etwa 80\% komplex gebunden (17); der Rest ist ionisiert und Bestandteil des intrazellulären Mg-Puffersystems (18), so daß die Konzentration des ionisierten $\mathrm{Mg}$ in der Zelle in einem gröBeren Bereich nahezu unabhängig vom Gesamt- $\mathrm{Mg}$ sein wird. Dieses Verhalten konnte bei $E$. coli direkt gemessen werden (1). Es ist also anzunehmen, $\mathrm{da} B$ in $\operatorname{der}$ tierischen Zelle bei der wesentlich geringeren Abnahme des $\mathrm{Mg}$-Gehaltes die intrazelluläre Mg-Ionenaktivität auch praktisch konstant bleibt und die intrazellulären $\mathrm{Mg}$-Ionen nicht im Diffusionsgleichgewicht mit den extrazellulären $\mathrm{Mg}$-Ionen stehen. Daraus ergibt sich die Frage, wie die Änderungen der Wasser-, Na- und KVerteilung zustande kommen.

Bei $E$. coli-Zellen wurde der verminderte aktive Transport $\mathrm{Mg}$-armer Zellen durch Zugabe von $\mathrm{Mg}$ nicht normalisiert, obgleich die Zellen innerhalb weniger Minuten den gleichen Mg-Gehalt wie die Kontrollzellen hatten. Der K-Transport normalisierte sich erst, wenn die Bakterien wachsen und Protein synthetisieren konnten (2). Offenbar mußte erst ein Enzym oder Protein gebildet werden, das während der Ausbildung des $\mathrm{Mg}$-Mangels nicht mehr in ausreichender Menge gebildet wurde. Geringe Änderungen der Enzymaktivitäten konnten wir auch bei tierischen Zellen feststellen (19), ob die verminderte Menge eines Proteins für den veränderten $\mathrm{Na}-\mathrm{K}$-Transport verantwortlich ist, ist nicht bekannt.

Bei Mg-verarmten E. coli-Zellen sind Glykolyse und oxydative Phosphorylierung vermindert (20). Es besteht daher zweitens die Möglichkeit, daß eine für den aktiven K-Transport erforderliche energiereiche Verbindung nicht in ausreichender Konzentration vorhanden ist. $\mathrm{Ob}$ dies auch für den verminderten aktiven Transport tierischer Zellen zutrifft, bleibt offen, da der Stoff wechsel $\mathrm{Mg}$-verarmter tierischer Zellen nicht untersucht ist. Herzmuskelsarcosomen aus $\mathrm{Mg}$-arm ernährten Ratten waren zwar in vitro entkoppelt (21), besonders bei $\mathrm{Mg}$-freier Inkubation (22). Die Abnahme des P/OQuotienten konnte aber von anderen Untersuchern nicht bestätigt werden (23).

Eine andere Möglichkeit wäre die Aktivitätsabnahme der am Na-K-Transport bei tierischen Zellen beteiligten $\mathrm{Mg}$-abhängigen $\mathrm{Na}-\mathrm{K}$-aktivierbaren ATPase der Zellmembran. Infolge der starken Abnahme der extrazellulären $\mathrm{Mg}$-Konzentration könnte das Enzym an der Außenseite der Zellmembran nicht mehr mit $\mathrm{Mg}$ gesättigt sein.

Außerdem sind wahrscheinlich Mg-Komplexe mit Bestandteilen der Zellmembran durch die starke Abnahme der extrazellulären $\mathrm{Mg}$-Konzentration dissoziiert. Dadurch könnten Eigenschaften der Zellmembran und z. B. ihre Permeabilität für Alkaliionen geändert sein. 


\section{Literatur}

1. GÜNTHER, TH. und F. DoRN, Zschr. Naturfor. $24 b, 713$ (1969). 2. GüNTHER, Th. und P. MARIss, Zschr. Naturfor. 236,334 (1968).3. MaCINTYRE, I. und D. Davidson, Biochem. J. 70, 456 (1958). 4. WHANG, R. und L. G. WELT, J. Clin. Invest. 42, 305 (1963). 5. Martindale, L. und F. W. Heaton, Biochem. J. 92, 119 (1964). - 6. Walser, M., Erg. Physiol 59.185 (1967). - 7. DulCE, H. J. und Th. GüNther, Naunyn-Schmiedebergs Arch. exper. Pathol. Pharmakol. 238, 368 (1960). - 8. GÜNTHER, TH. und CH. Alter, diese Z. 5,67 (1967). - 9. Siegmund, P. und H.-J. DulCE, Hoppe-Seyler's Z. physiol. Chemie 320, 149 (1960). - 10. MERKER, H. J. und Th. GüNTher, diese Z. 8, 71 (1970). - 11. FranzinIArmstrong, C., Fed. Proc. 23, 887 (1964). - 12. Forssmann, W. G. und L. Girardier, Zschr. Zellforsch. 72, 249 (1966). -
13. Hrlt, D. K., J. Physiol. 179, 368 (1965). - 14. Zadunatsky, J. A., J. Cell Biol. 31, C 11 (1966). - 15. GüntHer, TH.; C. GOECKE und J. WolfF, Naunyn-Schmiedebergs Arch. exper. Pathol. u. Pharmakol. 258, 280 (1967). - 16. Maurirnus, A und F. H. Epstein, J. Clin. Invest. 42, 208 (1963). - 17. GüNTHER, TH., Zschr. Naturfor. 22b, 149 (1967). - 18. GüNTHER, TH., Zschr. Naturfor. $21 b, 1174$ (1966). - 19. GüNTHER, TH., diese Z. 8, 69 (1970). - 20. GüNTHER, TH., und P. Mariss, Hoppe-Seyler's Z. physiol. Chemie 349, 623 (1968). - 21. Vitale, J. J., M. NAKAMURA und D. M. Hegsted, J. biol. Chemistry 228, 573 (1957). 22. Giorgio, J. D., J. J. Virale und E. E. Hellerstern, Biochem. J. 82, 184 (1962). - 23. BeECHY, R. B., N. Alcock, S. HANNA und I. MacInTYRE, Biochem. J. 71, 181 (1959).

Prof. Dr. Th. Günther 1000 Berlin 33, Arnimallee 22 